\title{
O Papel do Materialismo como Mediador entre o Prazer nas Compras e a Compra por Impulso*
}

\author{
Samuel Lincoln Bezerra Lins \\ Pontificia Universidade Católica do Rio de Janeiro \\ Rio de Janeiro, RJ, Brasil \\ Gabrielle Poeschl \\ Universidade do Porto \\ Porto, Portugal
}

\begin{abstract}
RESUMO
Muitas pesquisas foram realizadas sobre a compra por impulso, mas raros são os estudos com o público adolescente. O objetivo deste estudo foi testar um modelo de mediação do materialismo entre o prazer nas compras e a compra por impulso com adolescentes brasileiros $(n=482)$ e portugueses $(n=238)$, utilizando a técnica de modelagem de equações estruturais. Apesar de alguns problemas com o ajustamento do modelo de medida, o modelo apresentou bons índices de ajustamento para ambas as amostras, obtendo validade externa. Os resultados sugerem que os valores materialistas mediam a relação entre o prazer oriundo da atividade de compra e a compra por impulso, evidenciando a relação existente entre variáveis que foram estudadas separadamente em estudos prévios sobre a compra por impulso. Eles também constituem uma abordagem à problemática no Brasil e em Portugal em particular, e com o público adolescente, em geral.
\end{abstract}

Palavras-chave: Adolescentes; Compra por impulso; Mediação; Materialismo; Prazer.

\section{ABSTRACT}

\section{The Role of Materialism as a Mediator between Shopping Pleasure and Impulse Buying}

Much research has been carried out on impulse buying but studies with young people are scarce. The objective of this study was to assess whether the relationship between the pleasure to shop and impulse buying was mediated by materialism. It was conducted with Brazilian $(n=482)$ and Portuguese $(n=238)$ adolescents, employing structural equations modeling. In spite of a few adjustment problems with the measurement model, the goodness of fit of the mediation model was good with both samples, providing external validity. Results suggest that materialistic values mediate the relationship between the pleasure deriving from the activity of buying and impulse buying, highlighting the relationship that exists between variables that were separately tested in previous studies on impulse buying. They also constitute an approach to this issue in Brazil and Portugal in particular, and with the adolescent public, in general.

Keywords: Adolescents; Impulse buying; Mediation; Materialism; Pleasure.

\section{RESUMEN}

\section{El Papel del Materialismo como Mediador entre el Placer de Ir de Compras y la Compra por Impulso}

Muchas investigaciones se han realizado sobre la compra por impulso, pero pocos estudios con el público adolescente. El objetivo de este estudio fue probar un modelo de mediación del materialismo entre el placer de la compra y la compra por impulso con adolescentes brasileños $(n=482)$ y portugueses $(n=238)$, utilizando la técnica de modelos de ecuaciones estructurales. A pesar de algunos problemas con el ajuste del modelo de medición, el modelo mostró buenos índices de ajuste para ambas muestras, y la validez externa.Los resultados sugieren que los valores materialistas median la relación entre el placer derivado de la actividad de compra y la compra por impulso, que muestra la relación entre las variables que se estudiaron por separado en estudios previos sobre la compra por impulso. También representan un enfoque para el problema en Brasil y Portugal en particular, y con las audiencias adolescentes, en general.

Palabras clave: Adolescentes; Compra por impulso; Mediación; Materialismo; Placer.

* Esta pesquisa foi financiada pelo Programa Erasmus Mundus External Cooperation Window (Projecto MUNDUS17 - coordenado pela Universidade do Porto). 
Acompra por impulso tem sido uma área amplamente investigada nos últimos anos (Gąsiorowska, 2011; Kacen, Hess \& Walker, 2012; Lins \& Pereira, 2011; Masouleh, Pazhang \& Moradi, 2012; Muruganantham \& Bhakat, 2013; Tifferet \& Herstein, 2012; Verplanken \& Sato, 2011). Uma consulta realizada na base de dados EBSCO em Janeiro de 2014, com os termos "impulse buying", "impulse purchase" ou "impulse shopping" resultou em 1.251 publicações, e constatou-se um crescente número de estudos publicados nos últimos anos.

Entretanto, muitas das investigações sobre a compra impulsiva foram realizadas com adultos (Kacen, Hess \& Walker, 2012) ou com universitários (Lins \& Pereira, 2011) mas de forma surpreendente, poucos estudos focaram o público adolescente. Os estudos encontrados são raros e relativamente recentes. Temos conhecimento de apenas sete investigações sobre a compra por impulso em adolescentes. De forma sucinta, estes estudos revelaram que os adolescentes com perfis de compradores impulsivos tendem a comprar mais roupas do que os outros (Yu \& Seock, 2002) e passam mais tempo circulando na loja (Yaoyuneyong \& George, 2010). Os adolescentes com uma baixa inteligência emocional (Lin \& Chuang, 2005) e socialmente ansiosos (Lin \& Chen, 2012) também tendem a fazer mais compras impulsivas. Alguns autores encontraram uma relação positiva entre a tendência a idolatria (admiração e devoção excessiva a algo ou alguém) e a compra por impulso (Yang, Wang \& Niu, 2008), enquanto outros não verificaram a relação entre estas duas variáveis (Sharma, 2011). Constatouse ainda que a participação de adolescentes num curso sobre planeamento financeiro reduz a probabilidade de efectuarem compras por impulso (Lai, 2010).

Apesar de o mercado ter muitas vezes como alvo os adolescentes, na crença de que as pessoas mais jovens têm mais recursos financeiros disponíveis (Simpson, Douglas, \& Schimmel, 1998), pouca atenção foi dada aos adolescentes nas investigações (Santos \& Fernandes, 2011). Para além disso, poucas investigações sobre a compra por impulso foram realizadas no Brasil e em Portugal, e nenhuma com o público adolescente.

\section{A COMPRA POR IMPULSO}

De acordo com Arnould, Price e Zinkhan (2004), a compra por impulso ocorre quando os consumidores sentem uma vontade repentina, juntamente com um desejo emocional intenso de comprar imediatamente. Rook e Fisher (1995), por sua vez, definem a compra impulsiva como "a tendência do consumidor para comprar espontaneamente, sem reflexão, de forma imediata, estimulado pela proximidade física do objeto desejado, dominado pela atracção emocional e absorvido pela promessa de gratificação imediata" (p. 306).

Segundo Youn (2000) a compra por impulso é composta por duas dimensões: a dimensão afectiva e a dimensão cognitiva. A dimensão afectiva diz respeito às emoções e aos estados de humor, e é composta por três elementos: (1) irresistível desejo de comprar, (2) emoções positivas em relação à compra, e (3) gestão do humor. A dimensão cognitiva refere-se às estruturas mentais e processos envolvidos no pensamento, compreensão e interpretação. Esta dimensão inclui elementos como (1) a vontade de agir sem avaliar o custo-benefício, (2) a falta de um planeamento claro do que se deseja comprar, e (3) a despreocupação com as consequências do seu comportamento de compra.

A compra por impulso não deve ser considerada um fenômeno patológico, mas um hábito típico de consumo contemporâneo, especificamente do ambiente de retalho (Wood, 1998). Com efeito, a compra impulsiva diferencia-se da compra por compulsão. A compra compulsiva é caracterizada pela constante vontade de comprar, como forma principal de compensar uma ansiedade excessiva (Dittmar, 2005). Este tipo de compra é crónico e cíclico. O indivíduo comporta-se de maneira perturbada e tensa na hora da compra e, após o acto de compra, recai sobre ele um sentimento de culpa por agir desta maneira.

A principal diferença entre as duas formas de compra é que a compra por compulsão se refere ao comportamento de compra e a compra por impulso diz respeito ao produto a ser adquirido (Faber \& O'Guinn, 2008). Contudo, vários estudos mostram uma estreita relação entre os dois tipos de compra (Eren, Eroğlu \& Hacioglu, 2012). Das variáveis que apresentam uma forte relação com a compra por impulso podemos destacar o materialismo e o prazer nas compras.

\section{O MATERIALISMO}

O materialismo está presente no dia-a-dia dos consumidores (Richins \& Dawson, 1992). Ele prevalece na sociedade de consumo actual, e pode ser uma resposta à compreensão do fenômeno da compra impulsiva, por serem os dois constructos altamente correlacionados (Arocas, Arrieta, \& Botero, 2004).

Ao longo das últimas décadas, o materialismo tornou-se um dos valores pessoais que mais tem despertado interesse entre os estudiosos e investigadores. Richins e Dawson (1992) definem o materialismo como um valor pessoal que reflecte a importância que algumas pessoas dão à posse de bens 
materiais. Os autores defendem que o materialismo é composto por três dimensões: (a) Centralidade, que se refere à aquisição de bens ocupando um lugar central na vida das pessoas, isto é, adquirir bens como um estilo de vida; (b) Felicidade, que diz respeito à satisfação pessoal, ao alcance da felicidade por meio de aquisições; e (c) Sucesso, que faz referência à relação directa entre o acúmulo de bens e ao ser bem-sucedido, resumindo basicamente a ideia de que ter posses é ter sucesso.

A evidência empírica é consistente em relação ao facto de que o materialismo está positivamente associado com factores determinantes dos comportamentos desviantes de compra como a ansiedade, depressão, satisfação com a vida e impulsividade (Troisi, Christopher, \& Marek, 2006). Pessoas muito materialistas são menos satisfeitas, menos felizes, e têm mais problemas psicológicos (Dittmar, 2011).

A literatura científica corrobora que as motivações que originam o comportamento de compra por impulso estão profundamente enraizadas na adesão aos valores materialistas (Arocas et al., 2004). Neste sentido, é concebível sugerir que a compra impulsiva está associada com a adesão a valores materialistas (Kasser et al., 2007).

Lins e Pereira (2011) encontraram que o valor pessoal "Prazer" (desfrutar da vida, satisfazer todos os seus desejos) e "Estabilidade pessoal" (ter certeza que amanhã terá tudo o que tem hoje, ter uma vida organizada e planificada) estão relacionados positivamente com a compra impulsiva, ou seja, a valorização do hedonismo e a procura de uma vida financeiramente estável predizem o comportamento de compra impulsiva. Por sua vez, Dittmar (2005) mostrou que a adesão aos valores materialistas (traduzidos em afirmações como 'A minha vida seria melhor se eu tivesse certas coisas que não tenho' e 'Eu admiro pessoas que têm casas, carros e roupas caras') é um preditor significativo da compra impulsiva. Desta forma, o materialismo destaca-se como uma variável que apresenta uma estreita relação com a compra por impulso (Chavosh, Halimi, \& Namdar, 2011; Segal \& Podoshen, 2012), e é um dos valores que tem grande relevância nos estudos da psicologia do consumo (Richins \& Rudmin, 1994).

\section{O PRAZER NAS COMPRAS}

Hirschman e Holbrook (1982) destacaram a importância dos aspectos intangíveis e subjectivos que fazem parte do acto de consumo, como a fantasia, a experiência com o produto no ponto de venda, e os aspectos emocionais. Assim, os desejos de gratificação que o indivíduo sente ao adquirir um produto estão relacionados directamente com a compra impulsiva (Arocas et al., 2004), pois o prazer nas compras é talvez o factor mais fortemente associado com a compra por impulso (Činjarević, Tatić, \& Petrić, 2011; Verplanken $\&$ Sato, 2011). Deste modo, os consumidores que têm a maior probabilidade de realizarem compras impulsivas são aqueles que estão motivados por desejos hedônicos ou por razões não económicas, como a diversão e a satisfação social ou emocional (Hausman, 2000).

Além disso, os típicos compradores impulsivos costumam relatar elevados sentimentos de excitação e considerações hedonistas que acompanham a realização da compra impulsiva (Herabadi, Verplanken, \& van Knippenberg, 2009). Portanto, a impulsividade na compra também é resultante de motivações emocionais ou hedônicas (Babin, Darden \& Griffin, 1994).

Devido à grande oferta de produtos no mercado, os consumidores passaram a procurar algo mais no processo de consumo, procurando experiências diferenciadas, compostas de significados e valores que tenham relação com seu estilo de vida (Hirschman \& Holbrook, 1982). Pode-se dizer que Holbrook e Hirschman (1982) foram os pioneiros no estudo da "perspectiva experiencial" do consumo. Os autores questionaram a visão tradicional do marketing, que poderia negligenciar outros fenômenos presentes no consumo, como o lazer, prazeres sensoriais e respostas emocionais.

Assim, novas abordagens surgiram focando suas atenções na experiência do cliente, como forma de acrescentar valor ao produto, aproximando as experiências de consumo ao conceito de valor (Lusch, Vargo, \& O'Brien, 2007). A criação de valor é resultado da experiência individual de um consumidor em determinado contexto de compra (Prahalad \& Ramaswamy, 2004). Esta experiência pode ocorrer em diversos momentos da compra: quando está a procurar um produto, durante o processo de compra ou quando está a consumir o produto (Brakus, Schmitt, \& Zarantonello, 2009).

O prazer é talvez o factor que está mais associado à compra por impulso (Verplanken \& Sato, 2011). Comprar por impulso desempenha um importante papel na realização dos desejos hedônicos associados ao consumo hedônico. Este papel suporta uma ligação conceitual entre a motivação de compra hedônica e o comportamento de compra por impulso. Assim, os consumidores mais susceptíveis de se empenharem em compras impulsivas são aqueles que estão motivados por desejos hedonistas ou por razões não económicas, como a fantasia, diversão e satisfação social ou emocional (Hausman, 2000). 
As pessoas que vivenciam maior prazer nas compras também expressam mais atitudes materialistas, devido à difusão da crença de que a aquisição de bens materiais é a chave da felicidade e do bem-estar. A fomentação deste pensamento intensificou-se a partir do século XIX (Richins \& Dawson, 1992), estabelecendo a relação entre satisfação (prazer) e bem adquirido (produto material).

Diversos estudos apontam para a relação entre prazer nas compras e compra por impulso (Arocas, Arrieta \& Botero, 2004; Činjarević, Tatić, \& Petrić, 2011; Hausman, 2000; Herabadi, Verplanken, \& van Knippenberg, 2009; Verplanken \& Sato, 2011), entre o materialismo e a compra por impulso (Arocas, Arrieta, \& Botero, 2004; Segal \& Podoshen, 2012; Troisi, Christopher, \& Marek, 2006), e entre o prazer nas compras e o materialismo (Arocas, Arrieta, \& Botero, 2004; Eren, Eroğlu, \& Hacioglu, 2012). Deste modo, procurou-se testar um modelo de análise de mediação da relação entre o prazer (variável independente) e a impulsividade na compra (variável dependente), mediada pelo materialismo na compra (variável mediadora), conduzindo dois estudos. $\mathrm{O}$ primeiro estudo foi conduzido com adolescentes brasileiros e o segundo, visando testar a validade externa do modelo, ou seja, verificar a possibilidade de generalizar os resultados para outros grupos de pessoas, com adolescentes portugueses.

Escolheu-se de conduzir os estudos no Brasil e em Portugal, por serem dois países com grandes semelhanças históricas e culturais que, atualmente, vivem uma situação económica contrastada. Enquanto o Brasil está a experimentar um acelerado desenvolvimento econômico, Portugal tem vivido um período de intensa crise econômica com elevados índices de desemprego. Desta forma, considerámos relevante estudar este tipo de comportamento de consumo (compra por impulso) neste público-alvo (adolescentes) nestes dois países (Brasil e Portugal).

\section{MÉTODO}

\section{Amostra}

No primeiro estudo participaram 482 adolescentes brasileiros estudantes de escolas públicas e privadas (213 rapazes e 269 raparigas; idade média $=14.96$ anos, $D P=1.07)$ e no segundo 238 adolescentes portugueses de uma escola pública (117 rapazes e 121 raparigas; idade média $=15.43 ; D P=1.76)$.

\section{Instrumentos}

Primeiramente, os adolescentes responderam a questões sociodemográficas (sexo e idade), e depois responderam aos seguintes instrumentos:

\section{Impulso nas compras e \\ Prazer nas compras}

Tanto a escala de Impulso nas compras como a escala de Prazer nas compras foram retiradas do Consumer Style Inventory (CSI) (Sproles \& Kendall, 1986) adaptada para o público adolescente no contexto brasileiro (Santos \& Fernandes, 2006). Cada escala é composta por quatro itens $(1=$ discordo totalmente; $7=$ concordo totalmente)

\section{Materialismo}

A escala usada para medir a importância atribuída às posses pelo respondente $(1=$ discordo totalmente; 7 = concordo totalmente) é uma escala unidimensional composta por oito itens (Richins, 2004), amplamente utilizada em estudos transculturais (Schaefer, Hermans, \& Parker, 2004) e adaptada por Ponchio, Aranha e Todd (2007) para o contexto de consumidores brasileiros de baixo rendimento, obtendo assim uma melhor compreensão dos itens.

\section{Análise dos dados}

Para testar o modelo de mediação, utilizou-se a técnica de modelagem de equações estruturais (MES). A técnica de modelagem de equações estruturais é uma mescla da análise factorial e da análise de regressão, que possibilita testar estruturas factoriais de escalas de medida (análise factorial confirmatória), bem como analisar as relações explicativas entre diversas variáveis simultaneamente, sejam elas observadas ou latentes.

Esta técnica tem sido amplamente aplicada tanto no campo das Ciências Sociais e Humanas (Marôco, 2010), como na Psicologia (Pilati \& Laros, 2007), e nos estudos do comportamento do consumidor e no marketing (Priester, 2010). Acredita-se que esta técnica é melhor do que os testes que empregam a análise de regressão para avaliar modelos de mediação (Iacobucci, Saldanha, \& Deng, 2007).

O modelo de mediação do materialismo foi avaliado com o software AMOS (versão 19 do programa SPSS Statistical Package for the Social Sciences) utilizando o método da máxima verossimilhança. No ajustamento do modelo, utilizou-se a estratégia two-step: (1) o primeiro passo consistiu em ajustar o modelo de medida, e (2) o segundo passo foi efectuar o ajuste do modelo estrutural.

Também foi calculada a fiabilidade compósita $(F C)$ do instrumento. Considerou-se que $F C \geq .70$ indica uma fiabilidade de constructo adequada (Fornell \& Larcker, 1981). Neste sentido, também verificou-se o peso factorial dos itens (lambdas: $\lambda$ ). Todos os pesos 
factoriais devem ser estatisticamente significativos (Bagozzi \& Phillips, 1991), recomendando-se valores de lambda superiores a .50 (Johnson \& Stevens, 2001).

A existência de outliers foi avaliada pela distância quadrada de Mahalanobis $\left(D^{2}\right)$, e a normalidade das variáveis foi verificada pelos coeficientes de assimetria $(S k)$ e curtose $(K u)$. Valores de $|S k|<3$ e $|K u|<10$ indicariam graves violações à distribuição normal (Marôco, 2010). Na avaliação da qualidade do ajustamento do modelo foram utilizados os indicadores $\chi^{2}, \chi^{2} / g l, o$ CFI (Comparative Fit Index), o GFI (Goodness of Fit Index), o TLI (Tucker Lewis Index), e o RMSEA (Root Mean Square Error of Approximation).

$\mathrm{O} \chi^{2}$ indica um bom ajustamento do modelo quando o $\chi^{2}$ é estatisticamente não significativo $(p \geq .05)$ (Bagozzi \& Yi, 2012). Para o indicador $\chi^{2} / g l$ o limite máximo de aceitação é 3 (Iacobucci, 2010).

Os índices CFI, GFI, e $T L I$, quando apresentam valores superiores a .90 , são considerados indicadores adequados para um bom ajuste (Marôco, 2010). Segundo Ullman (2007), valores entre .90 e .95 são indicadores razoáveis e valores acima de .95 são considerados bons ajustes. Quando os valores dos indicadores estão entre .80 e .90 , isto significa que o modelo tem um ajustamento aceitável, mas que necessita de um melhor refinamento (Marôco, 2010).

Para Browne e Cudeck (1993), quando o RMSEA apresenta um coeficiente máximo igual a .08, isto significa uma adequação razoável, quando o valor é menor que .05 significa uma boa adequação do modelo. Outros autores também afirmam que .08 é o limite máximo aceitável (Hancock \& Freeman, 2001).

Para refinar o modelo, recorreu-se aos índices de modificação (IM) calculados pelo AMOS. Considerou-se que $I M>45(p<.001)$ apontavam para problemas de ajustamento local, e depois de avaliada a plausibilidade teórica das modificações, correlacionaram-se os erros de medida que conduziram à melhoria do ajustamento do modelo de medida.

Para verificar de forma mais precisa a significância estatística dos efeitos indirectos e dos efeitos totais, optou-se por utilizar o método de reamostragem Bootstrap para um conjunto de 5000 amostras aleatórias. $\mathrm{O}$ intervalo de confiança resultante deste método deve ser significativamente diferente de zero com $p<.001$.

Ainda foi realizado o teste de Bollen-Stine, que também é um método de simulação de amostras que verifica a significância estatística dos efeitos indirectos e totais. Para um bom ajustamento, este indicador deve ter o valor de $p<.001$.

Para a utilização da $M E S$, recomenda-se, pelo menos, 10 respondentes por variável (Hair et al., 2005). No nosso modelo, temos 4 variáveis da escala impulso nas compras, 4 variáveis da escala prazer nas compras, e mais 8 variáveis da escala materialismo, totalizado 16 variáveis. Portanto, as amostras desta investigação cumpriram esta exigência (Estudo 1 Brasil: $n=482$; Estudo 2 Portugal: $n=238$ ).

\section{Procedimentos}

Para realizar os nossos estudos fez-se, inicialmente, um contacto com escolas do ensino secundário, solicitando, através do envio de carta, a autorização para a aplicação do inquérito com os alunos. Após autorização, entrou-se em contacto com os professores para que os questionários pudessem ser aplicados durante as aulas. Os termos de consentimento livre e esclarecido foram obtidos e os instrumentos foram administrados em sala de aula. O projecto de pesquisa foi submetido e aprovado pela Comissão de Ética da Faculdade de Psicologia e Ciências das Educação (FPCEUP) da Universidade do Porto (Portugal).

\section{RESULTADOS}

\section{Análise factorial confirmatória dos instrumentos de medida - Brasil}

A escala que avalia o impulso nas compras apresenta índices de ajustamento muito bons, $\chi^{2}(2)=2.670$, $p=.263, \chi^{2} / g l=1.33, G F I=.997, C F I=.997, T L I=.991$, $R M S E A=.026$. A fiabilidade compósita obteve o valor ligeiramente insatisfatório de .61, mas todos os pesos factoriais, lambdas $(\lambda)$, foram estatisticamente significativos e diferentes de zero $(p<.001)$. Mesmo obtendo dois itens com pesos factoriais baixos (impulso1 e impulso4) optou-se por permanecer com a escala completa, pois a retirada dos itens não interferia significativamente no ajustamento do modelo.

A escala de prazer nas compras também apresentou índices de ajustamento muito bons, $\chi^{2}(2)=0.262$, $p=.877, \chi^{2} / g l=1.33, G F I=1.00, C F I=1.00, T L I=1.00$, $R M S E A=.065$. A fiabilidade compósita obteve o valor de .73 e todos os pesos factoriais $(\lambda)$ foram estatisticamente significativos $(p<.001)$. Dois itens (prazer3 e prazer4) apresentaram um peso factorial $<.50$, contudo, dado que sua retirada não interferia significativamente no ajustamento, optámos por utilizar a escala completa.

Por fim, a análise factorial confirmatória da escala materialismo também apresentou bons índices de ajustamento apesar de o valor do qui-quadrado ser significativo: $\chi^{2}(18)=5.47, p<.001, \chi^{2} / g l=3.03$, $G F I=.971, C F I=.969, T L I=.952, R M S E A=.065$. O facto do $\chi^{2}$ ter sido significativo, levar-nos-ia à rejeição do instrumento, no entanto este indicador é muito sensível ao tamanho da amostra e, por isso, devemos 
examinar outros índices (Bagozzi et al., 2012). Como os outros indicadores apresentaram bons índices de ajustamento, o modelo não foi rejeitado.

A escala apresentou fiabilidade compósita de .83, e todos os pesos factoriais foram estatisticamente significativos $(p<.001)$ e maiores que .50 . Para um melhor ajustamento do modelo, foram correlacionados os erros de medida dos itens materialismol e materialismo2, e materialismo3 e materialismo5. Mesmo com a identificação de alguns problemas no ajuste, os modelos de medida apresentaram bons índices de ajustamento, possibilitando a aceitação dos modelos de medida. Sendo assim, podemos passar para a análise da adequabilidade do modelo de mediação.

\section{Análise da adequabilidade do modelo de mediação - Brasil}

O modelo tem um ajustamento razoavelmente bom à estrutura de variância e covariância dos 16 itens analisados, $\chi^{2}(99)=263.41, p<.001, \chi^{2} / g l=2.66$, $G F I=.934, C F I=.929, T L I=.914, R M S E A=.059$. A percentagem da variância explicada pelo modelo é de 29\% (ver Figura 1). Para um melhor ajustamento do modelo, foram correlacionados os erros de medida dos itens materialismo1 e materialismo2, e materialismo3 e materialismo5.

Constata-se que há um efeito directo significativo do prazer nas compras sobre o materialismo, $\beta=.77 ; p<.001$, e um efeito total do prazer sobre a impulsividade, $\beta=.45 ; p<.001$, sendo que, quando o materialismo é inserido na equação, o prazer passa a exercer, sobre a impulsividade, um efeito directo menor e não significativo, $\beta=.08 ; p=.430$, enquanto que o efeito directo do materialismo sobre a impulsividade permanece significativo, $\beta=.48 ; p<.001$.

$\mathrm{O}$ efeito de mediação analisado é significativo $(p<.001)$ segundo o método Bootstrap de reamostragem. A estimativa do efeito indirecto do prazer sobre a impulsividade na compra está enquadrada por um intervalo de confiança a 90\% com limites de ].22; .52[, com o indicador Bollen-Stine de um bom ajustamento $(p<.001)$ aplicado em 5000 amostras. Deste modo, os resultados dão apoio à nossa predição de que o efeito de mediação do materialismo na relação prazerimpulsividade na compra existe.

\section{Análise factorial confirmatória dos instrumentos de medida - Portugal}

Inicialmente, verificámos os índices de ajustamento da escala impulso nas compras, $\chi^{2}(2)=0.515, p=.773$, $\chi^{2} / g l=.258, \quad G F I=.999, \quad C F I=1.000, \quad T L I=1.00$, $R M S E A<.000$, que nos permitem afirmar que o modelo é muito bom. Os pesos factoriais, lambdas $(\lambda)$, foram estatisticamente significativos e diferentes de zero $(p<.001 ; p<.006 ; p<.035)$, apesar de dois itens (impulso1 e impulso4) apresentarem pesos factoriais menores que .50 e a fiabilidade compósita

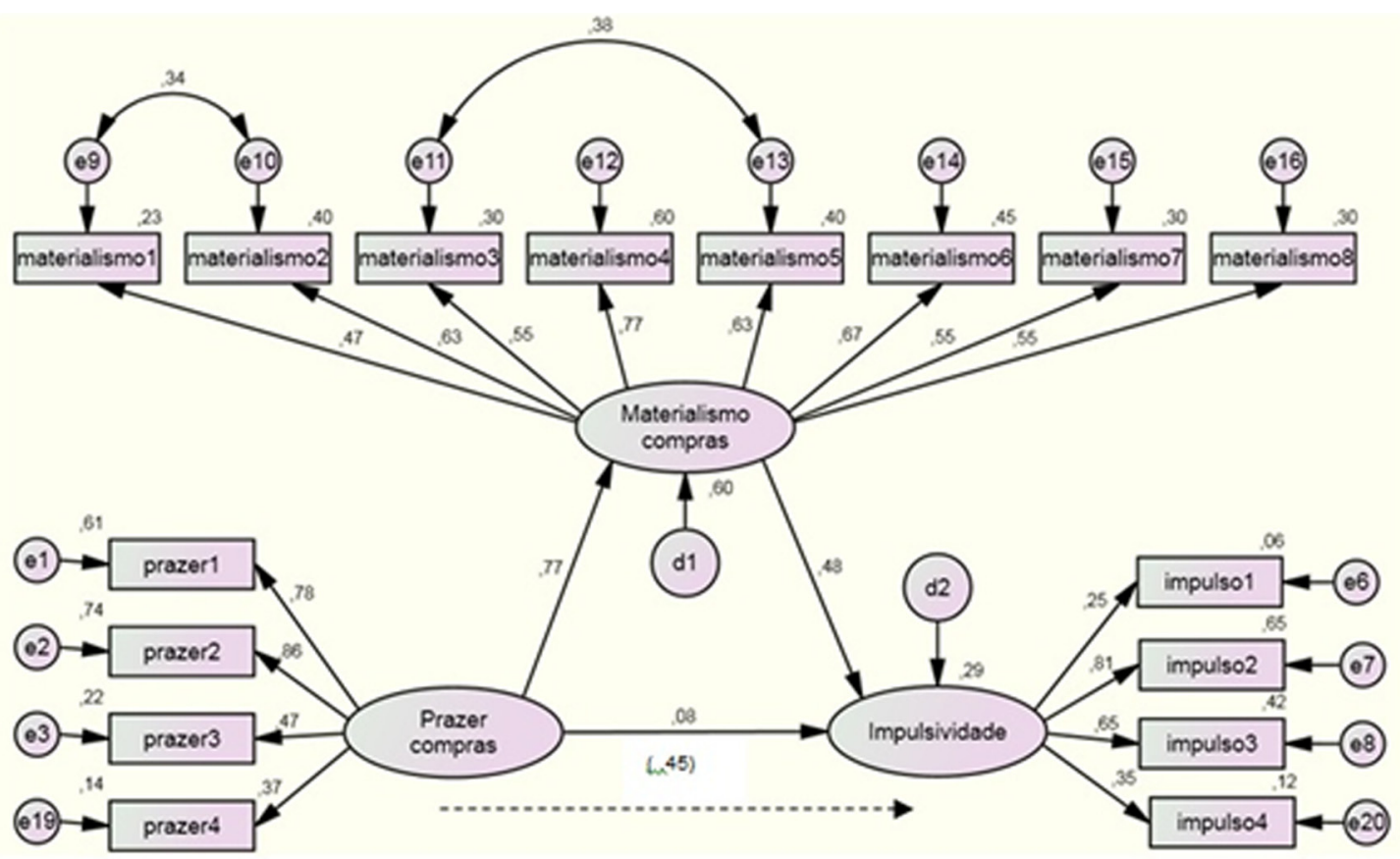

Figura 1. Modelo estrutural de mediação do materialismo entre o prazer e a impulsividade na compra - Brasil 
do modelo ter obtido de novo o valor pouco satisfatório de .59 .

A escala prazer nas compras também apresentou índices de ajustamento muito bons, $\chi^{2}(2)=3.12$, $p=210, \chi^{2} / g l=1.56, G F I=.993, C F I=.994, T L I=.983$, $R M S E A=.049$, comprovando a adequabilidade do modelo, com todos os pesos factoriais $(\lambda)$ estatisticamente significativos e diferentes de zero $(p<.001)$, apesar de dois itens (prazer4 e prazer3) terem pesos factoriais pouco adequados. A escala apresentou uma fiabilidade compósita .72 .

Por último, verificou-se os índices de ajustamento da escala materialismo, $\chi^{2}(18)=50.61, p<.001$, $\chi^{2} / g l=2.81, \quad G F I=.948, \quad C F I=.945, \quad T L I=.914$, RMSEA $=.087$, o que também comprovou a boa adequabilidade do modelo, mesmo se temos de novo um qui-quadrado significativo e um item com peso factorial abaixo do desejado. Para um melhor ajuste do modelo, foram correlacionados os erros de medida dos itens materialismo1 e materialismo2, e materialismo3 e materialismo5.

A fiabilidade compósita foi .83 e todos os pesos factoriais $(\lambda)$ foram estatisticamente significativos e maiores que .50 , com excepção do item "materialismo8" que obteve peso factorial .47. Apesar dos problemas verificados, os modelos de medida apresentaram bons índices de ajustamento dado que obtiveram $\chi^{2} / g l$ inferiores a 3, GFI, CFI e $T L I$ superiores a .90 .

\section{Análise da adequabilidade do modelo de mediação - Portugal}

O modelo tem ajustamento razoavelmente bom à estrutura de variância e covariância dos 16 itens analisados, $\chi^{2}(99)=241.32, p<.001, \chi^{2} / g l=2.44$, $G F I=.887, C F I=.872, T L I=.914, R M S E A=.078 . \mathrm{A}$ percentagem da variância explicada pelo modelo é de $23 \%$ (ver Figura 2). Para um melhor ajustamento do modelo, foram correlacionados os erros de medida dos itens materialismo1 e materialismo2, e materialismo3 e materialismo5.

Encontrou-se um efeito directo significativo entre o prazer nas compras e o materialismo, $\beta=.57 ; p<.001$, e um efeito total do prazer sobre a impulsividade, $\beta=.39 ; p<.006$, sendo que, quando o materialismo é inserido na equação, o prazer passa a exercer um efeito directo menor não significativo, $\beta=.10 ; p=.350$, e o efeito directo do materialismo sobre a impulsividade permanece significativo, $\beta=.52 ; p<.007$.

$\mathrm{O}$ efeito de mediação analisado é significativo $(p<.001)$, segundo o método Bootstrap de reamostragem. A estimativa do efeito indirecto do prazer sobre a impulsividade na compra está enquadrada por um intervalo de confiança a $90 \%$ com limites de ].19; .43[, com o indicador Bollen-Stine de um bom ajustamento $(p<.001)$ aplicado em 5000 amostras. Deste modo, os resultados permitem verificar que o efeito de mediação do materialismo na relação prazer-impulsividade existe.

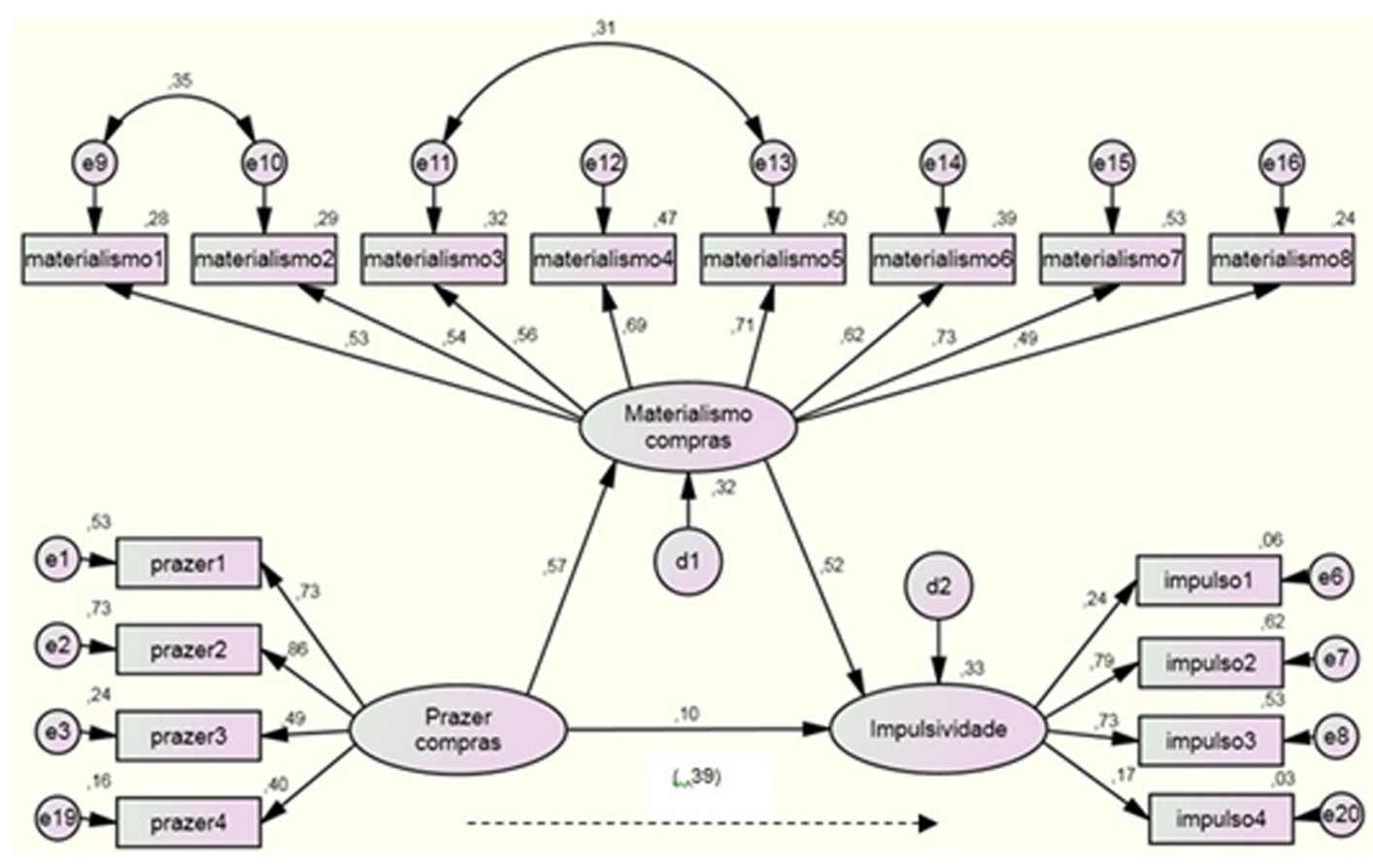

Figura 2. Modelo estrutural de mediação do materialismo entre o prazer e a impulsividade na compra - Portugal 


\section{DISCUSSÃO}

Em conformidade com o objetivo do nosso estudo, observamos que o modelo de mediação do materialismo entre o prazer nas compras e a impulsividade é verificado na amostra de adolescentes brasileiros, assim como na amostra de adolescentes portugueses. Assim, o prazer vivenciado na compra resulta na impulsividade na compra quando os adolescentes acreditam que a aquisição de bens materiais é, em conformidade com os estudos sobre o materialismo (Richins \& Dawson, 1992), a chave da felicidade e do sucesso.

Este modelo justifica as estratégias do marketing que tentam aumentar o tempo de envolvimento do consumidor na actividade de compra, procurando oferecer-lhe actividades interactivas, de lazer e de entretenimento. $\mathrm{O}$ aumento do envolvimento do consumidor dentro das lojas e dos centros comerciais pode resultar num aumento do dinheiro gasto e das intenções de compras futuras (Lueg et al., 2006). Algumas investigações também indicam que emoções positivas originadas num ambiente estimulante incentivam o consumidor a passar mais tempo dentro da loja (Hui \& Bateson, 1991) e, consequentemente, a realizar compras impulsivas. Sendo assim, o objetivo principal do mercado deveria ser, primeiramente, promover uma experiência agradável na actividade de compra.

O prazer que as pessoas sentem influenciaria positivamente a crença de que os bens materiais promovem a felicidade e o bem-estar, resultando na impulsividade na compra. Percebe-se que o foco de muitas estratégias de mercado visa proporcionar ao consumidor uma actividade de envolvimento de cunho emocional, que objetiva promover e intensificar a experiência positiva no acto da compra (Wakefield \& Baker, 1998). Diversos factores podem interferir na experiência de compra, como a interacção com o vendedor, o preço, a disponibilidade de recursos financeiros, a companhia de amigos (Jones et al., 2003), a memória e as atitudes do consumidor (Puccinelli et al., 2009), os afectos e o humor (Fiore \& Kim, 2007), a atmosfera da loja (Turley \& Milliman, 2000) e do centro comercial (Michon, Chebat, \& Turley, 2005).

Dado que, segundo a literatura revista, as pessoas materialistas tendem a ser mais insatisfeitas com a vida (Dittmar, 2011) e o consumo hedônico servir para melhorar o estado de humor (Hausman, 2000), poderíamos também esperar que o prazer nas compras atuasse como mediador entre o materialismo e a impulsividade. Este modelo alternativo também foi testado, contudo, não se verificou o efeito de mediação do prazer entre o materialismo e a impulsividade na compra.
Pelo contrário, o modelo que propomos apresentou índices de ajustamento aceitáveis, mas que aponta para a necessidade de um melhor refinamento. Isto aconteceu, possivelmente, devido aos problemas identificados no modelo de medida. Contudo, o modelo estrutural de mediação pôde ser verificado em dois contextos culturais, o que nos permite concluir que tem validade externa.

Cabe ressaltar que quatro erros do modelo foram correlacionados. Segundo Brown (2006), isto pode acontecer quando os itens têm conteúdos muito próximos, resultando na sua sobreposição. De fato, pode-se observar que há uma semelhança entre os itens correlacionados materialismo1 ("Admiro pessoas que possuem casas, carros e roupas caras") e materialismo 2 ("Gosto de gastar dinheiro com coisas caras"), e os itens materialismo3 ("Minha vida seria melhor se tivesse muitas coisas que não tenho") e materialismo5 ("Ficaria muito mais feliz se pudesse comprar mais coisas"). Os dois primeiros dizem respeito ao desejo de possuir coisas caras, e os dois últimos, à expectativa de que a compra torne a vida mais agradável.

Parece-nos que a relação entre variáveis podia se tornar mais clara se a impulsividade e o prazer nas compras tivessem sido avaliados com mais rigor. Com efeito, apesar do Consumer Style Inventory, de onde foram retiradas as escalas de impulso e prazer nas compras, ser um instrumento amplamente difundido (Azizi \& Makkizadeh, 2012; Jain \& Sharma, 2013), ainda carece de melhorias e aperfeiçoamento para poder avaliar melhor os constructos de impulsividade e prazer nas compras.

Deve-se acrescentar que, mesmo se a compra por impulso é intrinsecamente emocional, há também uma dimensão cognitiva (Youn, 2000). Esta dimensão não foi abordada no instrumento de medida destes estudos, pois os itens faziam referência predominantemente aos aspectos emocionais. Seria, portanto, interessante verificar se este modelo também se aplica à dimensão cognitiva da impulsividade da compra.

O modelo testado nos dois estudos apresentados é inovador por ser verificado empiricamente pela primeira vez. Trata-se de um modelo parcimonioso, por considerar apenas uma variável independente e uma variável mediadora ao contrário de outros modelos explicativos da compra por impulso que englobam mais de cinco variáveis (Beatty \& Ferrell, 1998; Cahyorini \& Rusfian, 2011; Harmancioglu, Finney, \& Joseph, 2009). Os nossos resultados permitem compreender melhor o fenômeno da compra por impulso nos dois países estudados, Brasil e Portugal, assim como no público adolescente, um público pouco estudado na investigação sobre a compra por impulso. 


\section{CONSIDERAÇÕES FINAIS}

Os resultados encontrados nesta investigação pretendem contribuir para o avanço do conhecimento acerca da compra por impulso nos dois países estudados, Brasil e Portugal. Pretendem também contribuir para a literatura científica internacional no âmbito da psicologia do consumidor, por ter utilizado como amostra o público adolescente, pouco estudado na investigação sobre a compra por impulso. O estudo deste público parece-nos particularmente importante dado que a actividade de compra faz parte da rotina de muitos adolescentes e que eles são alvo de estratégias de marketing cada vez mais agressivas para os levar a comprarem impulsivamente.

Este estudo procura também contribuir para o avanço do campo da psicologia social aplicada aos estudos do consumo, um campo que desafia a crítica muitas vezes endereçada à psicologia social de não ser suficientemente utilizada no cotidiano (Bagozzi, Gürhan-Canli, \& Priester, 2009). De fato, a psicologia social aplicada aos estudos do consumo é uma área em crescente desenvolvimento que integra diferentes teorias e métodos, com diferentes abordagens de investigação e prática.

As contribuições trazidas por este trabalho devem ser ponderadas pelas limitações que o cercaram. A primeira limitação refere-se à amostra utilizada, não probabilística, e a segunda ao fato de ter considerado que os adolescentes de todas as idades faziam parte de um único grupo homogêneo. Estudos apontam contudo que há diferenças dentro do período etário da adolescência (12 a 18 anos), no que diz respeito à socialização ao consumo e a aspectos como o materialismo e a autoestima. Os adolescentes mais novos (12-13 anos) diferenciam-se significativamente dos adolescentes mais velhos (17-18 anos) (Chaplin \& Roedder-John, 2007), mas este estudo não levou em conta estas diferenças. A terceira limitação provém de não ter sido considerados os aspectos cognitivos da compra por impulso. Seria relevante, em estudos futuros, utilizar instrumentos que considerem ambos os aspectos.

\section{REFERÊNCIAS}

Arnould, E., Price, L., \& Zinkhan, G. (2004). Consumers Boston: McGraw-Hill.

Arocas, R., Arrieta, S., \& Botero, M. (2004). La compra impulsiva y el materialismo en los jóvenes estudio exploratorio en estudiantes universitarios de Barranquilla (Colombia). Psicología desde el Caribe: Revista del Programa de Piscología de la Universidad del Norte, (14), 1-26.

Azizi, S. \& Makkizadeh, V. (2012). Consumer decision-making style: the case of iranian young consumers. Journal of Management Research, 4(2), 88-111. http://dx.doi.org/10.5296/jmr.v4i2.1222

Babin, B., Darden, W., \& Griffin, M. (1994). Work and/or fun: measuring hedonic and utilitarian shopping value. Journal of Consumer Research, 20(4), 644-656. http://dx.doi.org/10.1086/209376

Bagozzi, R., Gürhan-Canli, Z., \& Priester, J. (2009). The social psychology of consumer behavior. Buckingham: Open University Press.

Bagozzi, R. \& Phillips, L. (1991). Assessing construct validity in organizational research. Administrative Science Quarterly, 36, 421-458. http://dx.doi.org/10.2307/2393203

Bagozzi, R. \& Yi, Y. (2012). Specification, evaluation, and interpretation of structural equation models. Journal of the Academy of Marketing Science, 40(1), 8-34. http://dx.doi.org/10.1007/s11747-011-0278-x

Beatty, S. \& Ferrell, M. (1998). Impulse buying: modeling its precursors. Journal of Retailing, 74(2), 169-191. http://dx.doi. org/10.1016/S0022-4359(99)80092-X

Brakus, J., Schmitt, B., \& Zarantonello, L. (2009). Brand experience: What is it? How is it measured? Does it affect loyalty? Journal of Marketing, 73(3), 52-68. http://dx.doi.org/10.1509/jmkg.73.3.52

Brown, T. (2006). Confirmatory factor analysis for applied research. New York: The Guilford Press.

Browne, M . \& Cudeck, R. (1993). Alternative ways of assessing model fit. In K. Bollen \& L. Long (Eds.), Testing structural equation model (pp. 136-162). Beverlly Hills: Sage.

Cahyorini, A. \& Rusfian, E. (2011). The effect of packaging design on impulsive buying. Journal of Administrative Science \& Organization, 18(1), 11-21.

Chaplin, L. \& Roedder-John, D. (2007). Growing up in a material world: age differences in materialism in children and adolescents. Journal of Consumer Research, 34(4), 480-493. http://dx.doi.org/10.1086/518546

Chavosh, A., Halimi, A., \& Namdar, J. (2011). The contribution of product and consumer characteristics to consumer's impulse buying behaviour in Singapore. 2011 International Conference of Social Science and Humanity, 5, 248-252.

Činjarević, M., Tatić, K., \& Petrić, S. (2011). See it, like it, buy it! Hedonic shopping motivations and impulse buying. Economic Review - Journal of Economics and Business, 9(1).

Dittmar, H. (2005). Compulsive buying: a growing concern? An examination of gender, age, and endorsement of materialistic values as predictors. British Journal of Psychology, 96, 467-491. http://dx.doi.org/10.1348/000712605X53533

Dittmar, H. (2011). Consumer culture, identity and well-being: the search for the 'good life' and the 'body perfect'. New York: Psychology Press.

Eren, S., Eroğlu, F., \& Hacioglu, G. (2012). Compulsive buying tendencies through materialistic and hedonic values among college students in Turkey. Procedia - Social and Behavorial Sciences, 58, 1370-1377. doi: http://dx.doi.org/10.1016/ j.sbspro.2012.09.1121

Psico, Porto Alegre, PUCRS, v. 46, n. 1, pp. 57-67, jan.-mar. 2015 
Faber, R. \& O'Guinn, T. (2008). Compulsive buying. In C. Haugtvedt, P. Herr \& F. Kardes (Eds.), Handbook of Consumer Psychology (pp. 1039-1056). New York: Psychology Press.

Fiore, A. \& Kim, J. (2007). An integrative framework capturing experiential and utilitarian shopping experience. International Journal of Retail \& Distribution Management, 35(6), 421-442. http://dx.doi.org/10.1108/09590550710750313

Fornell, C. \& Larcker, D. (1981). Evaluating structural equation models with unobservable variables and measurement error. Journal of Marketing Research, 18(1), 39-50. http://dx.doi.org/10.2307/3151312

Gąsiorowska, A. (2011). Gender as a moderator of temperamental causes of impulse buying tendency. Journal of Customer Behaviour, 10(2), 119-142. http://dx.doi.org/10.1362/147539211X589546

Hair, J., Anderson, R., Tatham, R., \& Black, W. (2005). Análise multivariada de dados. Porto Alegre: Bookman.

Hancock, G. \& Freeman, M. (2001). Power and sample size for the root mean square error of approximation test of not close fit in structural equation modeling. Educational and Psychological Measurement, 61(5), 741-758. http://dx.doi. org/10.1177/00131640121971491

Harmancioglu, N., Finney, R., \& Joseph, M. (2009). Impulse purchase of new products: an empirical analysis. Journal of Product \& Brand Management, 18(1), 27-37. http://dx.doi.org/10.1108/10610420910933344

Hausman, A. (2000). A multi-method investigation of consumer motivations in impulse buying behavior. Journal of Consumer Marketing, 17(5), 403-426. http://dx.doi.org/10.1108/07363760010341045

Herabadi, A., Verplanken, B., \& van Knippenberg, A. (2009). Consumption experience of impulse buying in Indonesia: emotional arousal and hedonistic considerations. Asian Journal of Social Psychology, 12(1), 20-31. http://dx.doi.org/10.1111/j.1467839X.2008.01266.X

Hirschman, E. \& Holbrook, M. (1982). Hedonic consumption: emerging concepts, methods and propositions. Journal of Marketing, 46(3), 92-101. http://dx.doi.org/10.2307/1251707

Holbrook, M. \& Hirschman, E. (1982). The experiential aspects of consumption: consumer fantasies, feelings, and fun. Journal of Consumer Research, 9(2), 132-140. http://dx.doi.org/10.1086/208906

Hui, M. \& Bateson, J. (1991). Perceived control and consumer choice on the service experience. Journal of Consumer Research, 18(2), 174-185. http://dx.doi.org/10.1086/209250

Iacobucci, D. (2010). Structural equations modeling: fit indices, sample size, and advanced topics. Journal of Consumer Psychology, 20, 90-98. http://dx.doi.org/10.1016/j.jcps.2009.09.003

Iacobucci, D., Saldanha, N., \& Deng, X. (2007). A Meditation on mediation: evidence that structural equations models perform better than regressions. Journal of Consumer Psychology, 17(2), 139-153. http://dx.doi.org/10.1016/S1057-7408(07)70020-7

Jain, R. \& Sharma, A. (2013). A review on Sproles \& Kendall's consumer style inventory (CSI) for analyzing decision making styles of consumers. Indian Journal of Marketing, 43(3), 48-56.

Johnson, B. \& Stevens, J. (2001). Exploratory and confirmatory factor analysis of the school level environment questionnaire (SLEQ). International Journal of Learning Envrionments Research, 4(3), 325-344. http://dx.doi.org/10.1023/A:1014486821714

Jones, M., Reynolds, K., Weun, S., \& Beatty, S. (2003). The product-specific nature of impulse buying tendency. Journal of Business Research, 56(7), 505-511. http://dx.doi.org/10.1016/S0148-2963(01)00250-8

Kacen, J., Hess, J., \& Walker, D. (2012). Spontaneous selection: the influence of product and retailing factors on consumer impulse purchases. Journal of Retailing and Consumer Services, 19(6), 578-588. http://dx.doi.org/10.1016/j.jretconser.2012.07.003

Kasser, T., Cohn, S., Kanner, A., \& Ryan, R. (2007). Some costs of american corporate capitalism: a psychological exploration of value and goal conflicts. Psychological Inquiry, 18, 1-22. http://dx.doi.org/10.1080/10478400701386579

Lai, C. (2010). How financial attitudes and practices influence the impulsive buying behavior of college and university students. Social Behavior \& Personality: an International Journal, 38(3), 373-380. http://dx.doi.org/10.2224/sbp.2010.38.3.373

Lin, C. \& Chuang, S. (2005). The effect of individual differences on adolescents' impulsive buying behavior. Adolescence, 40(159), 551-558.

Lin, Y. \& Chen, C. (2012). Adolescents' impulse buying: susceptibility to interpersonal influence and fear of negative evaluation. Social Behavior and Personality, 40(3), 353-358. http://dx.doi.org/10.2224/sbp.2012.40.3.353

Lins, S. \& Pereira, R. (2011). Comprou sem querer? Entenda o porquê! RGBN - Revista Brasileira de Gestão e Negócios, 13(41), 376-395. http://dx.doi.org/10.7819/rbgn.v13i41.836

Lueg, J., Ponder, N., Beatty, S., \& Capella, M. (2006). Teenagers' use of alternative shopping channels: a consumer socialization perspective. Journal of Retailing, 82(2), 137-153. http://dx.doi.org/10.1016/j.jretai.2005.08.002

Lusch, R., Vargo, S., \& O'Brien, M. (2007). Competing through service: insights from service-dominant logic. Journal of Retailing, 83(1), 5-18. http://dx.doi.org/10.1016/j.jretai.2006.10.002

Marôco, J. (2010). Análise de equações estruturais, fundamentos teóricos, software \& aplicações. Pêro Pinheiro: Report Number.

Masouleh, S., Pazhang, M., \& Moradi, J. (2012). What is impulse buying? An analytical network processing framework for prioririzing factors affecting impulse buying. Management Science Letters, 2, 1053-1064. http://dx.doi.org/10.5267/ j.msl.2012.03.016

Michon, R., Chebat, J., \& Turley, L. (2005). Mall atmospherics: the interaction effects of the mall environment on shopping behavior. Journal of Business Research, 58(5), 576-583. http://dx.doi.org/10.1016/j.jbusres.2003.07.004

Muruganantham, G. \& Bhakat, R (2013). A review of impulse buying behavior. International Journal of Marketing Studies, 5(3), 149-160. http://dx.doi.org/10.5539/ijms.v5n3p149

Pilati, R. \& Laros, J. (2007). Modelos de equações estruturais em Psicologia: conceitos e aplicações. Psicologia: Teoria e Pesquisa, 23(2), 205-216. http://dx.doi.org/10.1590/S0102-37722007000200011

Ponchio, M., Aranha, F., \& Todd, S. (2007). Estudo exploratório do construto de materialismo no contexto de consumidores de baixa renda do município de São Paulo. Revistas Gerenciais, 6(1), 13-21. 
Prahalad, C. \& Ramaswamy, V. (2004). Co-creation experiences: the next practice in value creation. Journal of Interactive Marketing, 18(3), 5-14. http://dx.doi.org/10.1002/dir.20015

Priester, J. (2010). The use of structural equation models in Consumer Psychology: a methodological dialogue on its contributions, cautions, and concerns. Journal of Consumer Psychology, 20, 205-207. http://dx.doi.org/10.1016/j.jcps.2010.03.005

Puccinelli, N., Goodstein, R., Grewal, D., Price, R., Raghubir, P., \& Stewart, D. (2009). Customer experience management in retailing: Understanding the buying process. Journal of Retailing, 85(1), 15-30. http://dx.doi.org/10.1016/j.jretai.2008.11.003

Richins, M. (2004). The material values scale: measurement properties and development of a short form. Journal of Consumer Research, 31(1), 209-219. http://dx.doi.org/10.1086/383436

Richins, M. \& Dawson, S. (1992). A consumer values orientation for materialism and its measurement: scale development and validation. Journal of Consumer Research, 19(3), 303-316. http://dx.doi.org/10.1086/209304

Richins, M. \& Rudmin, F. (1994). Materialism and economic psychology. Journal of Economic Psychology, 15(2), $217-231$. http://dx.doi.org/10.1016/0167-4870(94)90001-9

Rook, D. \& Fisher, R. (1995). Normative influences on impulsive buying behavior. Journal of Consumer Research, 22 (3), $305-$ 313. http://dx.doi.org/10.1086/209452

Santos, C. \& Fernandes, D. (2006). Avaliação da escala consumer style inventory (CSI) no contexto brasileiro. RAM - Revista de Administração Mackenzie, 7(2), 11-38.

Santos, C. \& Fernandes, D. (2011). A socialização de consumo e a formação do materialismo entre os adolescentes. RAM-Revista de Administracao Mackenzie, 12(1), 169-203. http://dx.doi.org/10.1590/S1678-69712011000100007

Schaefer, A., Hermans, C., \& Parker, R. (2004). A cross-cultural exploration of materialism in adolescents. International Journal of Consumer Studies, 28(4), 399-411. http://dx.doi.org/10.1111/j.1470-6431.2004.00395.x

Segal, B. \& Podoshen, J. (2012). An examination of materialism, conspicuous consumption and gender differences. International Journal of Consumer Studies, 37, 189-198. http://dx.doi.org/10.1111/j.1470-6431.2012.01099.x

Sharma, R. (2011). An investigation into impulse buying behavior among Indian urban adolescents. Abhigyan, 28(4), 43-49.

Simpson, L., Douglas, S., \& Schimmel, J. (1998). Tween consumers: catalog clothing purchase behavior. Adolescence, 33(131), 637-644.

Sproles, G. \& Kendall, E. (1986). A methodology for profiling consumers' decision-making styles. Journal of Consumer Affairs, 20(2), 267-279. http://dx.doi.org/10.1111/j.1745-6606.1986.tb00382.x

Tifferet, S. \& Herstein, R. (2012). Gender differences in brand commitment, impulse buying, and hedonic consumption. Journal of Product \& Brand Management, 2l(3), 176-182. http://dx.doi.org/10.1108/10610421211228793

Troisi, J., Christopher, A., \& Marek, P. (2006). Materialism and money spending disposition as predictors of economic and personality. North American Journal of Psychology, 8(3), 421-436.

Turley, L. \& Milliman, R. (2000). Atmospheric effects on shopping behavior: A review of the experimental evidence. Journal of Business Research, 49(2), 193-211. http://dx.doi.org/10.1016/S0148-2963(99)00010-7

Ullman, J. B. (2007). Structural equation modeling. In B. Tabachnick \& L. Fidell (Eds.), Using multivariate statistics (5 ed.) (pp. 676-780). Boston: Pearson Education.

Verplanken, B. \& Sato, A. (2011). The psychology of impulse buying: an integrative self-regulation approach. Journal of Consumer Policy, 34(2), 197-210. http://dx.doi.org/10.1007/s10603-011-9158-5

Wakefield, K. \& Baker, J. (1998). Excitement at the mall: determinants and effects on shopping response. Journal of Retailing, 74(4), 515-539. http://dx.doi.org/10.1016/S0022-4359(99)80106-7

Wood, M. (1998). Socio-economic status, delay of gratification, and impulse buying. Journal of Economic Psychology, 19(3), 295-320. http://dx.doi.org/10.1016/S0167-4870(98)00009-9

Yang, C., Wang, Y., \& Niu, H. (2008). The effects of idolatry and personality traits on impulse buying: an empirical study. International Journal of Management, 25(4), 633-640.

Yaoyuneyong, G. \& George, B. (2010). Impulse buying of apparel products: a study conducted among midwestern teenagers in the United States. Journal of Marketing Trends, 2(1), 5-20.

Youn, S. (2000). The dimensional structure of consumer buying impulsivity: measurement and validation. (Doctoral Thesis), University of Minnesota, Minneapolis.

Yu, J. \& Seock, Y. (2002). Adolescents' clothing purchase motivations, information sources, and store selection criteria: a comparison of male/female and impulse/nonimpulse shoppers. Family and Consumer Sciences Research Journal, 31(1), 50-77. http://dx.doi.org/10.1177/1077727X02031001003

Autores:

Samuel Lincoln Bezerra Lins - Doutor em Psicologia (Universidade do Porto), Pós-doutorando em Psicologia, Pontifícia Universidade Católica do Rio de Janeiro.

GABRIELle POESCHL - Professora associada de Psicologia, Universidade do Porto.

Endereço para correspondência:

Samuel Lincoln Bezerra Lins

Pontifícia Universidade Católica do Rio de Janeiro

Departamento de Psicologia

Rua Marquês de São Vicente, 225 - Gávea

22543-900 Rio de Janeiro, RJ, Brasil 